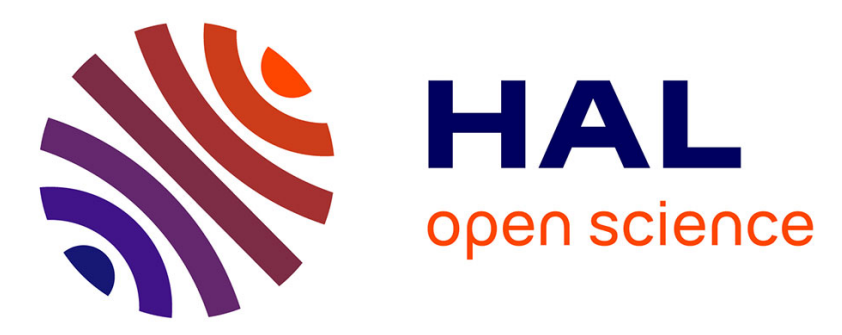

\title{
Vers une gestion sédimentaire durable de l'aménagement hydro-électrique Arc-Isère
}

F. Lauters, E. Laperrousaz, B. Camenen, J. Le Coz, F. Thollet, J. Némery, M. Delinares

\section{- To cite this version:}

F. Lauters, E. Laperrousaz, B. Camenen, J. Le Coz, F. Thollet, et al.. Vers une gestion sédimentaire durable de l'aménagement hydro-électrique Arc-Isère. La Houille Blanche - Revue internationale de l'eau, 2012, 1, p. 19 - p. 25. 10.1051/lhb/2012003 . hal-00761580

\section{HAL Id: hal-00761580 \\ https://hal.science/hal-00761580}

Submitted on 5 Dec 2012

HAL is a multi-disciplinary open access archive for the deposit and dissemination of scientific research documents, whether they are published or not. The documents may come from teaching and research institutions in France or abroad, or from public or private research centers.
L'archive ouverte pluridisciplinaire HAL, est destinée au dépôt et à la diffusion de documents scientifiques de niveau recherche, publiés ou non, émanant des établissements d'enseignement et de recherche français ou étrangers, des laboratoires publics ou privés. 


\title{
Vers une gestion sédimentaire durable de l'aménagement hydro-électrique Arc-Isère
}

\author{
Francois LAUTERS, Eric LAPERROUSAZ ${ }^{1}$, Benoît CAMENEN, Jérôme LE COZ, \\ Fabien THOLLET ${ }^{2}$, Julien NÉMERY ${ }^{1,3}$, Matthieu DELINARES ${ }^{4}$
}

\author{
1. EDF Tél : +33 (0)4 762024 13, Fax : +33 (0)4 762021 39-mail : Francois.lauters@edf.fr, eric.laperrousaz@edf.fr \\ 2. Cemagref - Unité de Recherche Hydrologie - Hydraulique, \\ Tél : +33 (0)4 722087 87,Fax : +33(0)4 78477875 e-mail : webmaster@lyon.cemagref.fr \\ 3. LTHE - Université de Grenoble 1/Grenoble-Inp, \\ Tél : +33 (0) 4766355 39, Fax : +33 (0) 4768250 14-mail : julien.nemery@gmail.com \\ 4. Artelia Eau Environnement \\ Tél : +33 (0)4 763343 91, Fax : +33 (0)4 76334212 -mail : matthieu.delinares@sogreah.fr
}

\begin{abstract}
RÉSUMÉ. - Le projet «gestion sédimentaire durable de la chaîne d'aménagements hydroélectriques Arc-Isère » a été mis en place par EDF suite au constat d'une sédimentation importante dans les bassins de cet aménagement, avec pour conséquence une moins bonne optimisation de la production. Ce projet intègre les principes de la méthodologie d'approche de la gestion sédimentaire sur les retenues appelée "état cible », intégrant les contraintes de nature sédimentaire, environnementale, et d'exploitation des aménagements. La première phase du projet présentée ici consiste en l'acquisition de connaissances nouvelles concernant les apports et transferts de sédiments fins au niveau des ouvrages et du cours d'eau, l'évolution morphologique des cours d'eau, les sédiments présents dans les retenues, le fonctionnement hydro-sédimentaire des aménagements et l'aspect économique lié aux sédiments. L'ampleur des mesures et études réalisées ou en cours, dont certaines sont encore du domaine de la recherche, met en évidence le besoin d'acquérir un volume important de connaissances pour définir une gestion sédimentaire durable qui réponde à des attentes en terme de production, d'équilibre économique et de contraintes environnementales et réglementaires.
\end{abstract}

Mots clefs : sédiment, Arc Isère, aménagement hydro-électrique, gestion sédimentaire

\section{Towards a sustainable management of sediment in the hydroelectric Arc-Isère facility}

ABSTRACT. - The project "Sustainable Management of Sediment in the hydroelectric Arc-Isère facility" has been set up by EDF to address productivity losses due to high sedimentation in the reservoirs of the development. This project uses the principles of the "target state " methodology. This approach to sediment management in reservoirs takes account of sedimentary, environmental, and operating factors. The first phase of the project presented here involves the acquisition of new knowledge in the following areas : the contributions and transfers of sediment in the works and rivers, the morphological evolution of the rivers, the sediments in the reservoirs, the hydro-sedimentary interactions and the economic impact of sediment. Our ultimate goal is to define a sustainable strategy that will address all aspects of sediment management including economics, production, environmental and regulatory constraints. In order to define this strategy our studies (some of them still in the research phase) indicate that large amounts of pertinent data will be required.

Key words : sediment, Arc Isère, Hydroelectric facility, sediment management

\section{INTRODUCTION : LE PROJET}

La chaîne d'aménagements hydroélectriques ArcIsère (figure 1) dérive l'eau captée sur l'Arc (retenue de St-Martin-la-Porte) vers l'Isère. L'eau captée est d'abord dirigée vers l'usine d'Hermillon puis est restituée dans le bassin de Longefan. Ce dernier assure un rôle de décantation des sédiments fins et de stockage à court terme et alimente, via une galerie sous le massif de Belledonne, le bassin du Flumet. Une prise secondaire dérive les eaux du Glandon, affluent de l'Arc, dans la galerie sous Belledonne. Le bassin du Flumet est le bassin amont de la Station de Transfert d'Energie par Pompage (STEP) du Cheylas qui restitue ses eaux au bassin aval du Cheylas puis à l'Isère.
L'Arc et le Glandon sont deux cours d'eau à fort transport solide (sédiments fins et grossiers). La gestion des prises d'eau en rivières par chasses hydrauliques et ouvertures lors des crues permet leur maintien dans un état d'engravement satisfaisant pour l'exploitation. Les sédiments fins captés avec l'eau entonnée par ces prises d'eau vont par contre en partie s'accumuler dans les bassins, réduisant d'autant leur volume utile et les performances à long terme de l'aménagement.

Devant ce constat, et dans le contexte nouveau des exigences de la DCE en termes de transport solide dans les cours d'eau et de développement durable, EDF a engagé une démarche visant à mieux prendre en compte la composante sédimentaire dans la gestion de 


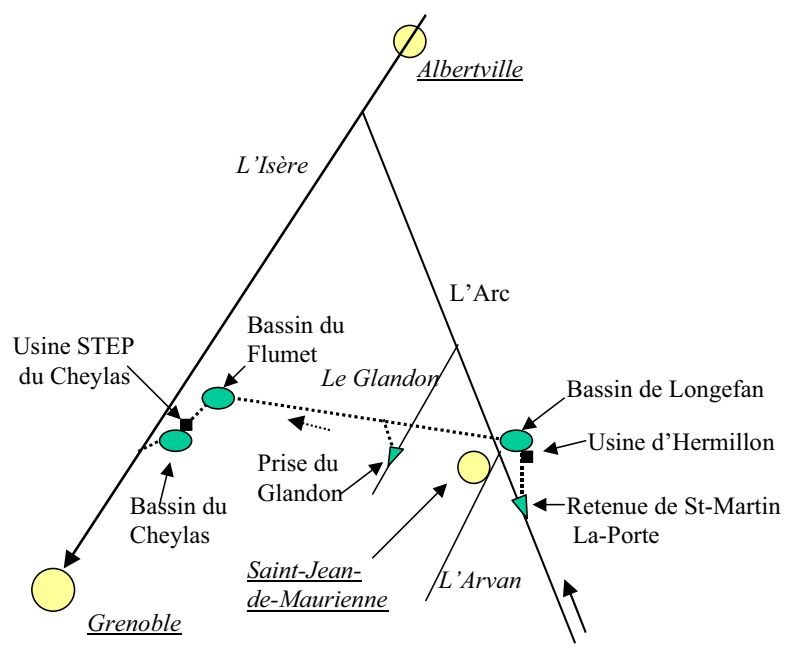

Figure 1 : Schéma de l'aménagement Arc-Isère.

l'aménagement. Il s'agit de définir de nouvelles modalités d'exploitation permettant de limiter les entrées de sédiments dans la chaîne en dérivation et de réduire et contenir le niveau d'envasement des bassins (contraintes d'exploitation, chasses et dragages...).

Ces objectifs du projet « gestion sédimentaire durable de l'aménagement Arc-Isère » imposent d'acquérir une connaissance suffisante sur :

- les cours d'eau concernant le transport solide et les évolutions morphologiques,

- les aménagements concernant les sédiments présents, les apports solides et leur transfert, et leur fonctionnement hydro-sédimentaire,

- les aspects économiques liés aux sédiments.

\section{ACQUISITION DE CONNAISSANCES SUR LES COURS D'EAU}

\section{II.1. Transport solide en suspension dans les cours d'eau}

Un réseau de 7 stations de mesure du transport solide en suspension par turbidimétrie, implantées sur les cours d'eau (2 sur l'Arvan, 1 sur le Glandon, 2 sur l'Arc et 2 sur
l'Isère), est exploité en commun par le Cemagref de Lyon, le LTHE de Grenoble, co-animateurs du site atelier Arc-Isère de la Zone Atelier Bassin du Rhône (ZABR), et EDF/DTG. Il est complété par la réalisation de mesures ponctuelles (mesures suite à des prélèvements ou à mise en place de stations temporaires) lors d'événements spécifiques comme des crues, des chasses de barrages ou des curages. Ce réseau doit permettre de caractériser les flux sédimentaires au droit des prises d'eau et de suivre le transit des matières en suspension (M.E.S.) dans les cours d'eau lors des événements de fort transport solide, qu'ils soient naturels où liés à la gestion des ouvrages.

En terme de connaissance des flux des cours d'eau, les chroniques sont pour l'instant trop courtes pour pouvoir en faire une analyse statistique permettant de définir les grandes caractéristiques du transport solide (flux annuel moyen, répartition annuelle des flux, courbes classées des teneurs de MES et de flux...) comme cela a déjà été fait à la station de Grenoble sur l'Isère [Mano 2008]. Toutefois, l'analyse des chroniques déjà disponibles montre des résultats très intéressants. Par exemple, sur une station mise en place temporairement sur l'Arc en amont du bassin de Longefan en 2010 (figure 2), il a été constaté entre mai et juillet des périodes avec des teneurs de M.E.S de plusieurs dizaines de $\mathrm{g} / \mathrm{l}$ lors de la crue de fin mai, lors de la chasse des retenues de l'Arc (8 juin) et lors d'épisodes de laves torrentielles sur des affluents (fin juin). Cela conduit sur ces trois mois à un flux solide cumulé estimé à plus de 400000 t. De tels résultats permettent de relativiser les apports liés aux événements d'exploitation particuliers (chasses et curages) par rapport aux événements naturels (crues, orages et fonte nivale) et laissent espérer qu'à l'avenir, l'évacuation régulière des dépôts qui se font dans la retenue de Longefan puisse être acceptable pour le cours d'eau.

Le Cemagref/Irstea [Camenen 2008] a déjà réalisé un premier travail de modélisation analytique pour estimer l'évolution d'un "nuage" de M.E.S le long de l'Arc au cours d'une chasse effectuée annuellement (figure 3). Il a ainsi permis de simuler le transfert du flux de matières en suspension engendré par des chasses du barrage de SaintMartin-la-Porte jusqu'à Grenoble. Les termes déjà obtenus par ce premier travail en ce qui concerne la vitesse de propagation et la diffusion pourront être utiles à la construction d'un modèle numérique plus complet prenant en compte les données en cours d'acquisition (thèse en cours sur ce sujet).

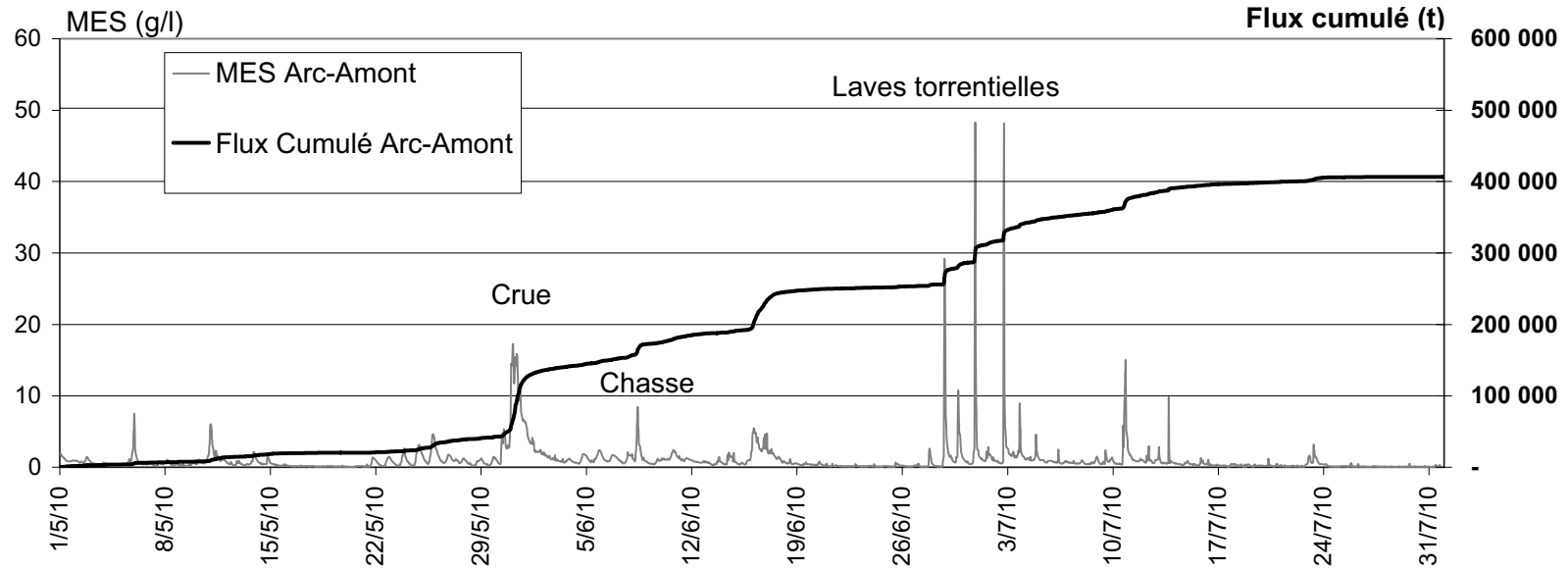

Figure 2 : Mesures de M.E.S et de Flux solide réalisées en 2010 sur l'Arc en amont de Longefan. 


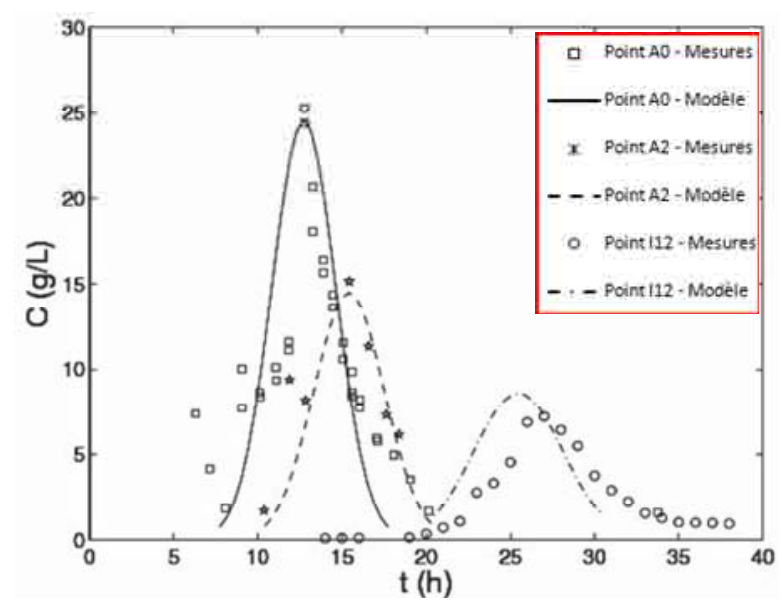

Figure 3 : Dynamique temporelle des M.E.S. mesurées et simulées au niveau de 3 points du réseau hydrographique par le modèle analytique Cemagref.

\section{II.2. Evolution morphologique des cours d'eau}

L'acceptabilité d'une modification de la gestion sédimentaire des aménagements doit passer par une analyse des effets possibles sur la morphologie de l'Arc et de l'Isère. Ces deux cours d'eau connaissent aujourd'hui des situations différentes. L'Arc, bien qu'endigué et soumis à des modifications des conditions hydrologiques liées à la présence des aménagements hydroélectriques, conserve une dynamique importante. L'Isère, sur certains tronçons, a au contraire une dynamique beaucoup plus faible avec notamment des successions de bancs latéraux formés de dépôts de sédiments fins fixés par la végétation. Ces bancs, qui réduisent la section d'écoulement et augmentent les risques d'inondation lors des fortes crues, ont récemment conduit le gestionnaire du cours d'eau à mettre en œuvre un programme de curage.

Un programme de suivi et d'étude de la morphologie des cours d'eau, mené en partenariat entre le Cemagref de Lyon et EDF a été mis en place. Il concerne le suivi d'un certain nombre de bancs de graviers caractéristiques de l'Arc et l'Isère, avec pour objectif de suivre la dynamique de ces bancs et notamment d'évaluer l'éventuelle formation de dépôts de sédiments fins, potentiellement amplifiés en cas de végétalisation des bancs. Ce suivi concerne la réalisation de profils en travers sur les bancs avec une description fine des substrats et notamment une évaluation de l'épaisseur des dépôts de sédiments fins (figure 4 et 5). Ces suivis sont réalisés de façon à cerner l'effet d'actions de gestion mises en place en test (chasses du bassin de Longefan) ou de travaux déjà programmés (curages). Dans le même cadre, un suivi LIDAR (topographie laser par moyen aéroporté) du lit de l'Arc et de l'Isère est programmé pour suivre les évolutions à grande échelle et disposer d'une donnée topographique complète des cours d'eau.

Enfin, le Cemagref poursuit les études sur un banc de graviers de l'Arc suivi depuis plusieurs années [Jodeau 2007]. Il s'agit au travers de plusieurs moyens d'investigation de comprendre l'évolution précise d'un banc en fonction des événements (figure 6).

Ainsi, l'évolution morphologique des cours d'eau est appréhendée à plusieurs échelles (de la rivière au grain). Toutes ces données seront utilisées pour la mise en place de modèles d'évolution du lit permettant de simuler les effets de modifications de gestion (thèse en cours sur ce sujet).
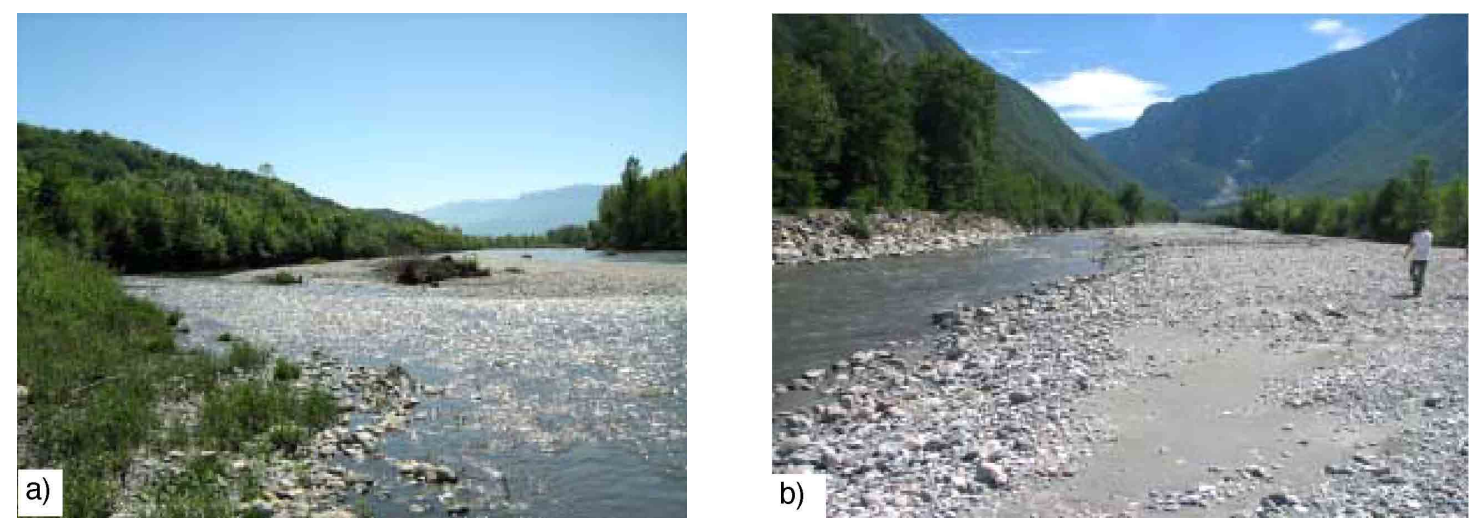

Figure 4 : Bancs de galets suivis sur l'Arc a) et l'Isère b).

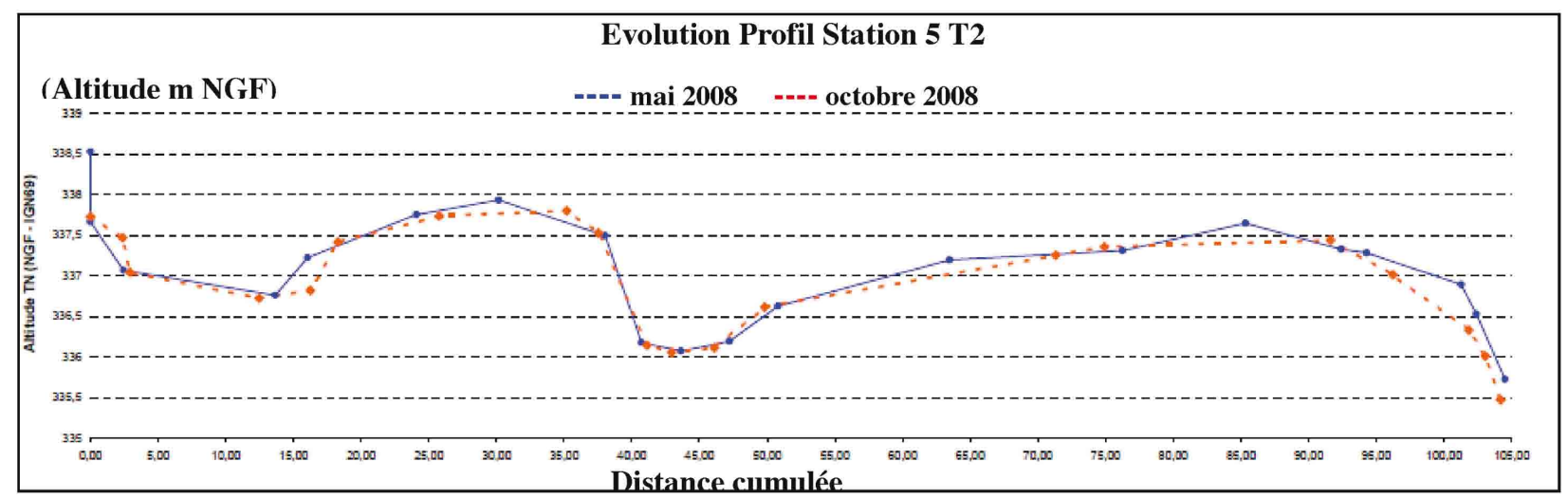

Figure 5 : Suivi d'un profil en travers sur un banc de galets de l'Arc 


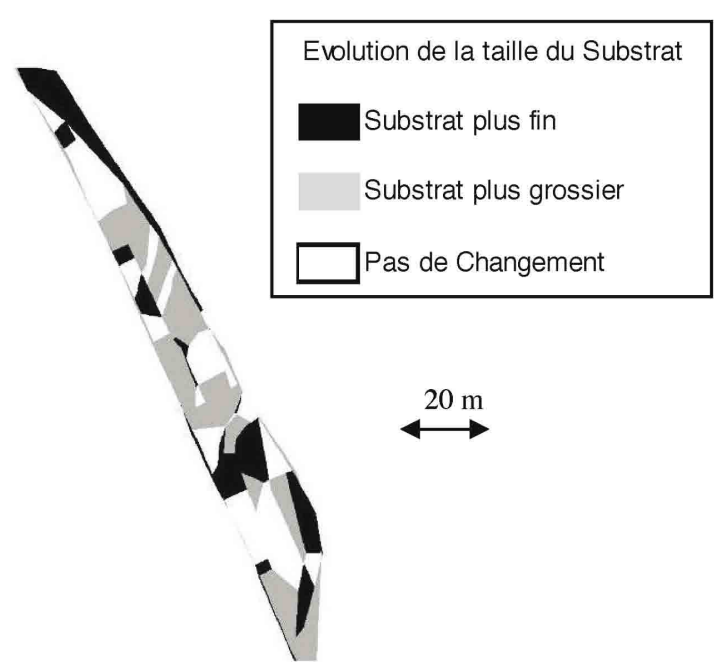

Figure 6 : Comparaison des zones de dépôts observées avant et après la chasse de juin 2006 sur le banc de graviers suivi par le Cemagref à St-Marie-de-Cuines (Arc en amont immédiat de la confluence avec le Glandon - cf figure 1).

\section{ACQUISITION DE CONNAISSANCES SUR LES AMÉNAGEMENTS}

\section{III.1. Les sédiments présents dans les retenues}

La connaissance des dépôts dans les bassins et de leur évolution en exploitation normale ou lors d'actions d'exploitation spécifiques (curages, chasses...) est obtenue par bathymétries régulières (figure 7). Ces éléments sont notamment utiles aux modélisations hydro-sédimentaires (cf III.3.) et à l'analyse historique de la sédimentation.

L'aspect qualité chimique des sédiments a été abordé dans un premier temps pour les sédiments du bassin de Longefan, sachant que ceux des autres bassins ont peu de chance d'être très différents. De nombreux prélèvements en surface et en profondeur par carottage ont été réalisés et analysés pour caractériser les différentes couches de sédiments sur l'ensemble de la surface. Les analyses ont porté sur les paramètres classiques (azotes...), sur les polluants suivis dans le cadre de la DCE, sur les PCB. Des analyses par tests de lixiviation ont également été réalisées. Ce suivi assez lourd se justifiait notamment dans l'optique d'une évacuation de la totalité des sédiments du bassin (éléments à fournir à l'Administration) avec une éventuelle valorisation de ces derniers ou un rejet dans le milieu. L'ensemble de ces

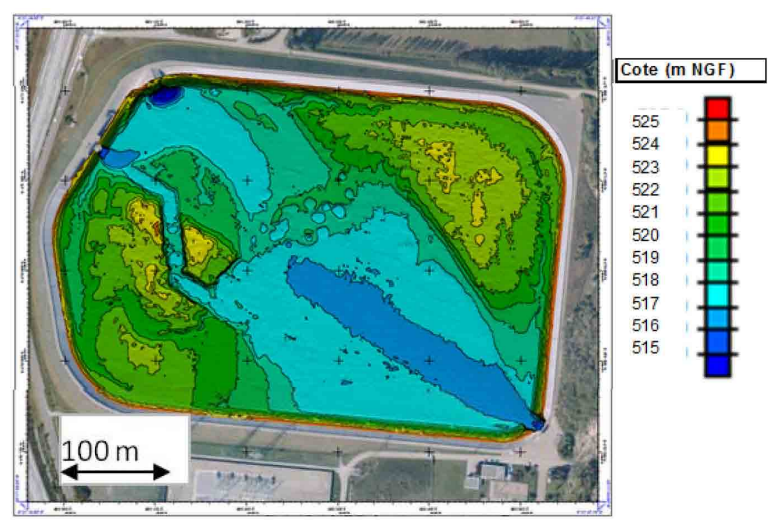

Figure 7 : Bathymétrie de la retenue de Longefan en mai 2009.

mesures n'a révélé aucune contrainte dans le cadre d'une manipulation des sédiments.

Enfin, afin de définir les caractéristiques physiques des sédiments, plusieurs types d'essais ont été réalisés sur les sédiments en place où sur des prélèvements intacts ou remaniés (tableau 1). Des essais par Jet Erosion Test (quantification des paramètres d'érosion par essais hydrauliques) ont été réalisés permettant de définir un coefficient d'érodabilité. Ces données sur les caractéristiques physiques sont exploitées dans le cadre des modélisations hydro-sédimentaires dans le bassin de Longefan (cf chapitre III.3).

\section{III.2. Apports et transferts de M.E.S au niveau des ouvrages}

Cet aspect est abordé au travers de la mise en place d'un réseau de stations de mesure en continu du transport solide en suspension. Cinq stations sont positionnées de façon à pouvoir connaître les entrées et sorties dans chaque bassin en termes de teneurs en M.E.S. mais aussi de flux solides. Quatre ans de données sont aujourd'hui disponibles. L'obtention de chroniques suffisamment longues est nécessaire pour prendre en compte la forte variabilité interannuelle concernant les apports par les cours d'eau. Il ressort de l'analyse de ces données un certain nombre d'informations qui permettent de mieux évaluer les apports et de mieux comprendre la dynamique (dépôts, reprises, transferts des M.E.S. dans les aménagements).

Il a été par exemple constaté des apports de M.E.S. vers le Flumet via la prise du Glandon et via l'Arc assez proches (tableau 2) alors que le flux liquide venant de la prise du

Tableau 1 : Limites d'Atteberg mesurées sur les sédiments du bassin de Longefan.

\begin{tabular}{|l|c|c|c|c|}
\hline & Carottes 1 & Carottes 2 & Carottes 3 & Carottes 4 \\
\hline Limite de Liquidité W1 & 31 & 25 & 20 & 28 \\
\hline Limite de Plasticité Wp & 23 & 22 & 19 & 24 \\
\hline Indice de Plasticité Ip & 8 & 3 & 1.5 & 4 \\
\hline
\end{tabular}

Tableau 2 : Valeurs observées en 2008 au niveau des deux entrées vers le bassin du Flumet.

\begin{tabular}{|l|c|c|}
\hline & Teneurs moyennes en MES (mg/l) & Flux en MES (\% du total entrant) \\
\hline Prise du Glandon & 380 & $45 \%$ \\
\hline $\begin{array}{l}\text { Bassin de Longefan - } \\
\text { départ vers la galerie de Belledonne }\end{array}$ & 50 & $55 \%$ \\
\hline
\end{tabular}


Glandon ne représente qu'environ $10 \%$ du flux liquide total. Cela traduit une charge en M.E.S bien plus importante de l'eau du Glandon que de l'eau arrivant de l'Arc après passage dans Longefan. Ce constat a conduit à mener une réflexion sur une possible modification de la gestion de la prise du Glandon.

Concernant la décantation dans le bassin de Longefan, la comparaison des flux entrant et sortant indique une décantation en moyenne de l'ordre de $10 \%$ sur l'année (données 2007-2008). Ce chiffre est faible dans la mesure où l'un des rôles de ce bassin est d'assurer la décantation des eaux de l'Arc avant le départ vers le Flumet. Il s'explique par l'envasement actuel du bassin et par une très forte hétérogénéité dans le temps, liée principalement au mode d'exploitation $\mathrm{du}$ bassin. Globalement, deux modes d'exploitation doivent être distingués : une exploitation à cote haute lorsque les débits entrant dans le bassin sont élevés en permanence et une exploitation qui alterne une cote moyenne avec une cote basse lorsque les débits entrant dans le bassin sont plus faibles.

La figure 8 montre, mois par mois pour l'année 2008, le pourcentage de stockage par rapport aux entrants en fonction de la cote du bassin (en fait le \% de temps avec une cote basse inférieure à $518.5 \mathrm{~m} \mathrm{NGF}$ ). On constate qu'en exploitation à cote haute quasiment constante (juin et juillet), le taux de stockage est de l'ordre de $40 \%$. En exploitation à cote basse, il y a, au contraire, une reprise dans le stock de sédiments en place. L'analyse des chroniques de M.E.S. en entrée et sortie du bassin a permis de confirmer qu'à cote basse, les vitesses d'écoulement dans le bassin sont telles qu'elles permettent l'érosion des sédiments.

Concernant les flux apportés par l'Arc dans le bassin de Longefan, ces derniers apparaissent faibles au regard des flux totaux sur l'Arc. Cela s'explique par le fait que sur l'Arc, l'essentiel du flux solide passe au moment des crues, périodes où les consignes de fonctionnement de l'aménagement conduisent à ne plus dériver l'eau de l'Arc depuis St-Martin-La-Porte.

\section{III.3. Caractéristiques hydro-sédimentaires des bassins usiniers}

Plusieurs études de modélisation hydraulique et hydrosédimentaire ont été menées par EDF/CIH et par SOGREAH à partir de codes de calcul du système TELEMAC.

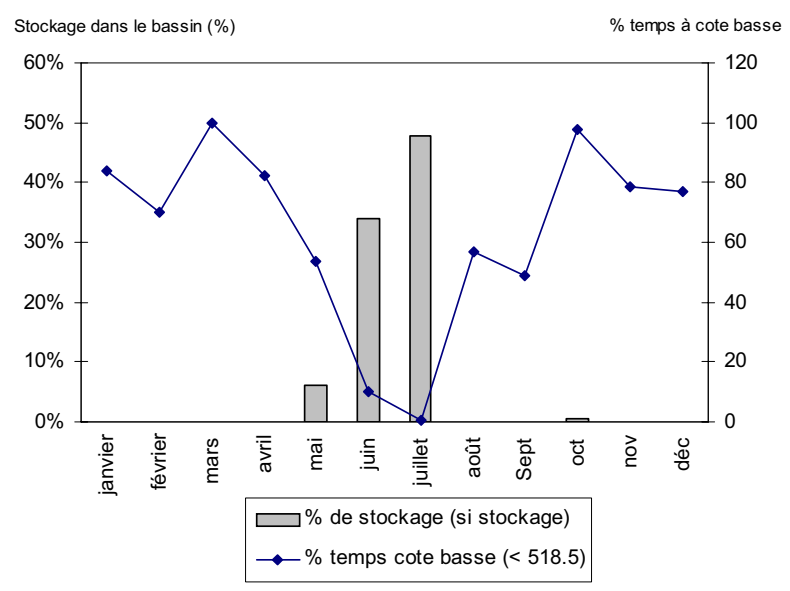

Figure 8 : Bilan du stockage dans le bassin de Longefan mois par mois en 2008 en fonction de la gestion.

La première étude avait pour objectif de définir les caractéristiques d'un reprofilage, par terrassement, des sédiments dans la retenue permettant d'accroître les vitesses d'écoulement lors d'une chasse et d'augmenter d'autant l'efficacité de celle-ci (figure 9). La seconde étude était du même type et visait à préparer une autre chasse avec un dragage préalable. Elle bénéficiait pour le calage hydraulique des résultats de la chasse précédente pendant laquelle avait été réalisées des mesures de vitesse par analyse d'images " Large Scale Particle Image Velocimetry » [Dramais 2010] et des relevés de ligne d'eau. Pour cette étude, le modèle TELEMAC 2D est couplé avec le code de transport sédimentaire SISYPHE et le calage sédimentaire tente de reproduire l'évolution des fonds de la précédente chasse.

Cette étude, bien que présentant encore beaucoup d'incertitudes, a permis la définition d'un programme de chasse prenant en compte le creusement préalable d'un chenal et la mise en place d'un déflecteur pour optimiser les vitesses dans le chenal créé et ainsi remplir les objectifs en terme de volume de sédiments érodés tout en maîtrisant les teneurs en MES restituées à l'Arc (contraintes environnementales).

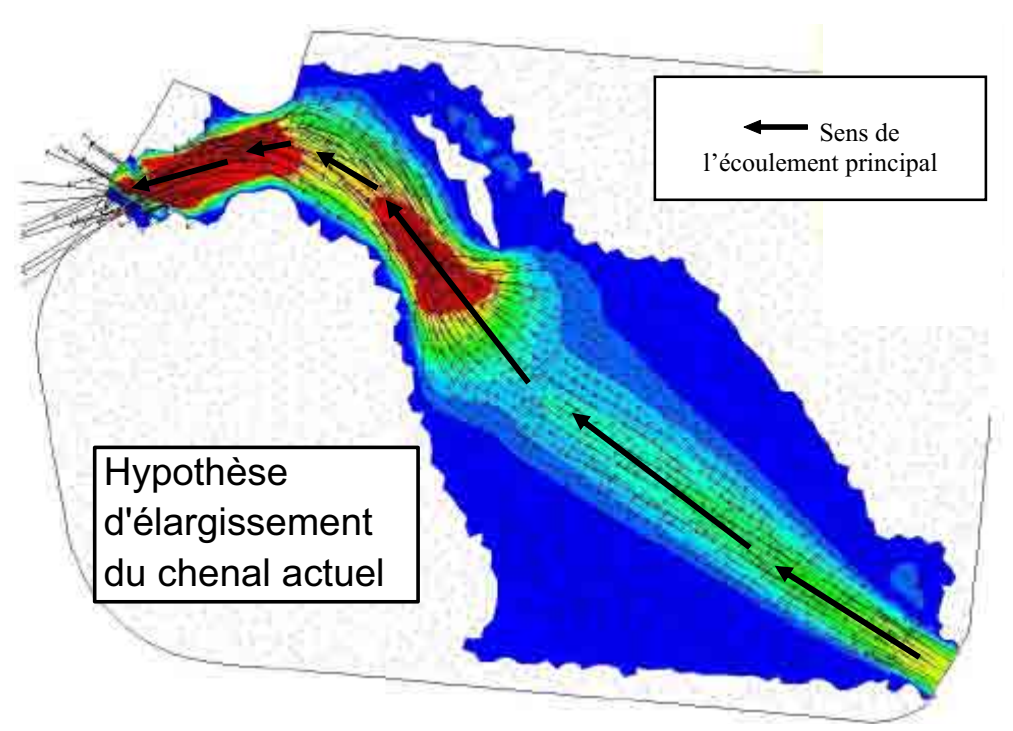

\begin{tabular}{|l|}
\hline \multicolumn{2}{|c|}{ Vitesse $(\mathrm{m} / \mathrm{s})$} \\
\hline 0 à 0.1 \\
\hline 0.1 à 0.2 \\
\hline 0.2 à 0.3 \\
\hline 0.3 à 0.4 \\
\hline 0.4 à 0.5 \\
\hline 0.5 à 0.6 \\
\hline 0.6 à 0.7 \\
\hline 0.7 à 0.8 \\
\hline 0.8 à 0.9 \\
\hline 0.9 à 1 \\
\hline
\end{tabular}

Figure 9 : Simulation lors d'une chasse avec un débit de $35 \mathrm{~m}^{3} / \mathrm{s}$. 
La troisième étude, avec le code hydro-sédimentaire tridimensionnel TELEMAC 3D, avait pour objectif la compréhension du fonctionnement hydro-sédimentaire du bassin à cote haute. Le calage hydraulique se base sur des mesures de vitesses par ADCP. Ces mesures ont été essentielles car elles ont mis en évidence une circulation du courant particulière avec une rotation dans le sens horaire le long des berges (figure 10). Le calage sédimentaire est réalisé en utilisant des mesures de M.E.S en entrée et sortie du bassin ainsi que les données d'un profil vertical de MES fait lors des mesures hydrauliques tandis que le calage morphologique a vérifié la capacité du modèle a reproduire les évolutions observées à long terme des formes des dépôts dans le bassin. L'exploitation du modèle, qui présente encore certaines incertitudes sur la reproduction des effets des événements courts (arrêts et reprises de turbinage), a notamment permis de donner une première estimation des gains attendus en terme de décantation par un curage complet du bassin (figure 11), et de tester l'impact de diverses propositions d'aménagement sur la capacité globale de décantation du bassin.

\section{IV. ÉTUDES ÉCONOMIQUES}

Les études économiques permettent de mettre en balance le gain de production associé aux apports liquides avec le passif correspondant aux apports solides et à leur sédimentation dans les bassins (exploitation et travaux de curage). Par exemple, une étude économique a été réalisée suite au constat que la prise secondaire du Glandon apportait vers le Flumet de l'ordre de $50 \%$ des sédiments mais seulement $10 \%$ du flux liquide. Il apparaissait en effet qu'une première action de gestion pouvait être rapidement mise en place en limitant les apports par le Glandon si cela se révélait justifié économiquement. Pour cette étude, le modèle PARSIFAL a été utilisé. Les données prises en compte sont les chroniques horaires de MES et de débit (2006-2008) au niveau des aménagements, un certain nombre d'hypothèses de valorisation économique, des chroniques hydrologiques et d'exploitation des aménagements et enfin plusieurs capacités du Flumet en fonction des dépôts. Cela a finalement conduit à proposer un seuil de teneur en MES au-delà duquel la prise du Glandon est mise hors d'eau certains mois de l'année.

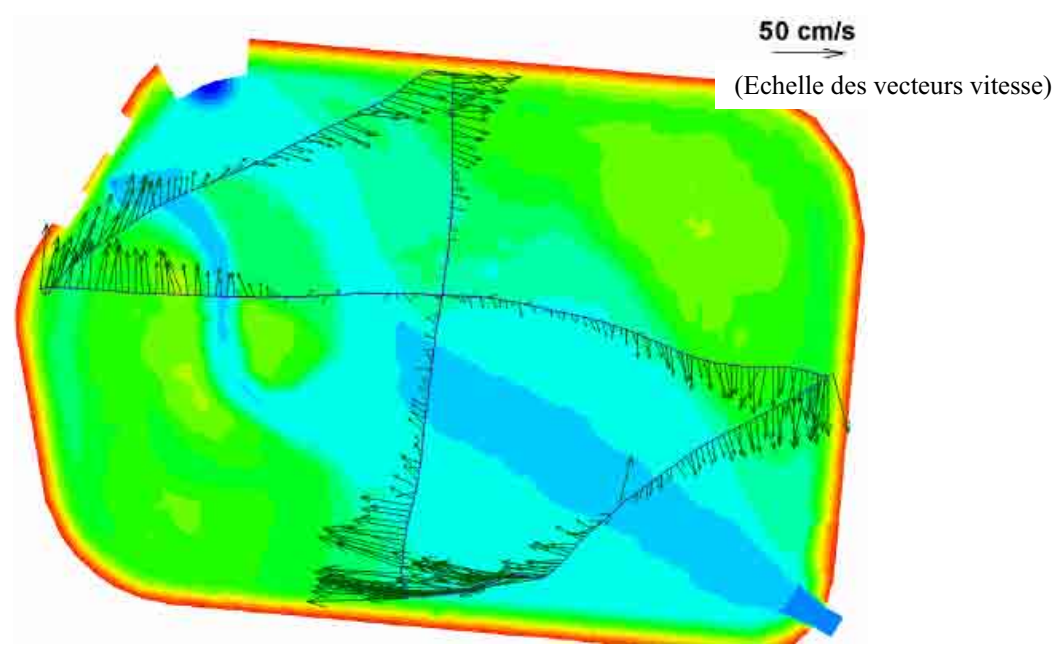

Figure 10 : Courants mesurés sur des profils dans le bassin de Longefan. Sur le fond de plan, en vert les massifs de sédiments, en bleu les zones sans sédiments.

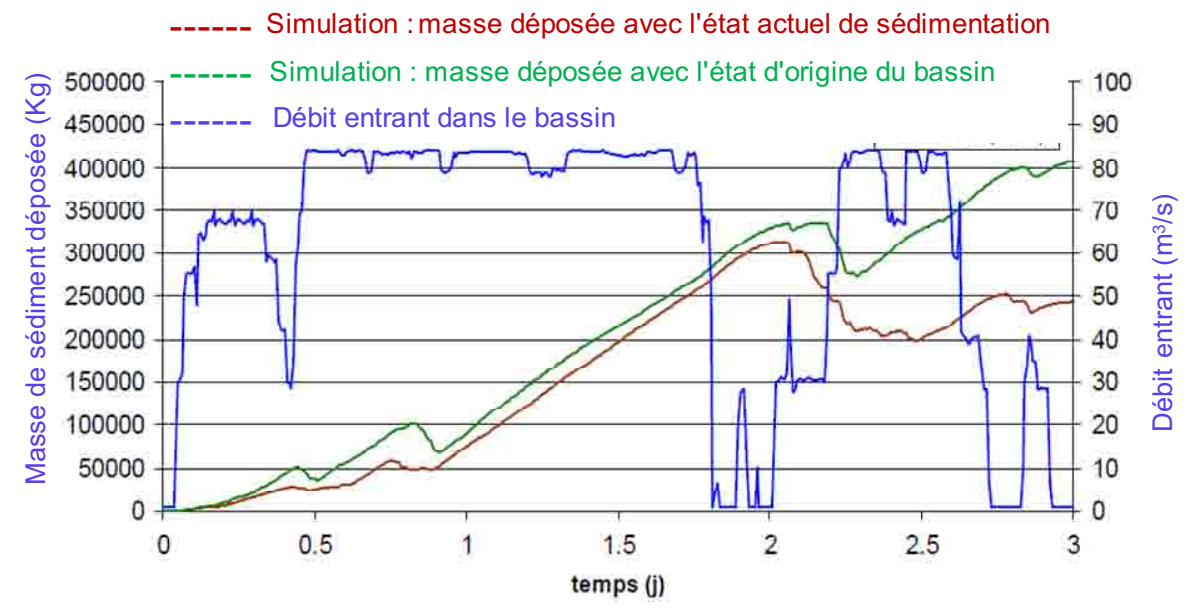

Figure 11 : Simulation de l'évolution temporelle des dépôts pour un scénario de gestion et d'apport de M.E.S. dans l'état actuel du bassin et dans l'état initial (bassin vide de sédiments). 


\section{CONCLUSION}

La définition globale d'une nouvelle gestion de l'aménagement sera établie une fois l'acquisition des connaissances terminée et l'ensemble des informations prises en compte. Cependant, les connaissances obtenues ont déjà conduit aux actions suivantes :

- mise hors d'eau de la prise du Glandon en fonction des teneurs en MES, permettant la limitation des apports vers le Flumet. Les résultats de l'étude économique ont en effet montré le bien-fondé de cette gestion comme le laissait supposer les données sur le transport solide.

- curage de l'essentiel du volume des sédiments du bassin de Longefan avec rejet à l'Arc, permettant de retrouver une capacité de décantation pour «protéger les bassins aval ».

\section{RÉFÉRENCES ET CITATIONS}

Camenen B., Jodeau J., \& Le Coz J. (2008) - Modélisation du flux sédimentaire pendant une chasse hydraulique. La Houille Blanche 04 : 83-89

Dramais G., Le Coz J., Camenen B., Hauet A. (2010) Advantages of a mobile LSPIV method for measuring flood discharges and improving stage-discharge curves. Journal of hydroEnvironmental Research (Submitted, Special issue on LSPIV)

Jodeau M. (2007) - Morphodynamique d'un banc de galets en rivière aménagée lors de crues. Thèse de doctorat, Université Claude Bernard, Lyon I

Mano V. (2008) - Processus conditionnant les apports de sédiments fins dans les retenues : optimisation des méthodes d'estimation des flux et modélisation statistique. Thèse de doctorat, Université Joseph Fourrier, Grenoble I. 312 p 